hep-th/0510195

\title{
D1-D5 on ALE Space
}

\author{
Kazumi Okuyama \\ Department of Physics and Astronomy, University of British Columbia \\ Vancouver, BC, V6T 1Z1, Canada \\ kazumi@phas.ubc.ca
}

We construct a two-dimensional $\mathcal{N}=(0,4)$ quiver gauge theory on D1-brane probing D5-branes on ALE space, and study its IR behavior. This can be thought of as a gauged linear sigma model for the NS5-branes on ALE space.

October 2005 


\section{Introduction}

The D1-D5-KK system (KK = Kaluza-Klein monopole) is a 1/8 BPS configuration in type IIB string theory. We are interested in the two-dimensional $\mathcal{N}=(0,4)$ gauge theory on the D1-brane of this system. In the limit where the KK-monopole is replaced by the orbifold $\mathbb{C}^{2} / \mathbb{Z}_{n}$, it is straightforward to construct the theory on D1-brane following the standard procedure of Douglas and Moore [1]. Namely, we consider the $\mathbb{Z}_{n}$ orbifolding of the $\mathcal{N}=(4,4) U\left(Q_{1}\right)$ gauge theory with one adjoint and $Q_{5}$ fundamental hypermultiplets coming from the D1-D5 system. The resulting theory is an $\mathcal{N}=(0,4)$ quiver gauge theory. As compared to the study of gauge theory of the D1-D5 system [2 [6], the $\mathcal{N}=(0,4)$ gauge theory of the D1-D5-KK system is less understood. See [7 11] for some of the works related to this system.

The D1-D5-KK system is related to the various brane configurations by duality. Obviously it is S-dual to the F1-NS5-KK system, and it is also dual to the triple intersection of M5-branes 12,13]. In general, $\mathcal{N}=(0,4)$ CFTs appear in many places in string theory; some of these are related to the D1-D5-KK system and some are not. The examples of $(0,4)$ preserving configuration that are not directly related to the D1-D5-KK system include the D1-D5-D9 system described by the $(0,4)$ ADHM sigma model [14,15, and the intersecting brane configuration of D1-D5-D5 [16].

One of the motivation to study the $\mathcal{N}=(0,4)$ quiver gauge theory is that it can be thought of as a gauged linear sigma model (GLSM) for the NS5-branes on ALE space. In general, GLSM is a quite useful tool to understand the moduli space of CFTs and the relations among them [17]. In [18,19,20], NS5-branes on $\mathbf{S}^{1}$ and their relation to the T-dual KK-monopoles are studied by using an $\mathcal{N}=(4,4)$ GLSM. It is very interesting to find GLSMs describing NS5-branes in other backgrounds. Our $\mathcal{N}=(0,4)$ quiver gauge theory is such an example.

This paper is organized as follows. In section 2, we construct the $\mathcal{N}=(0,4)$ quiver gauge theory describing the D1-D5 branes on $\mathbb{C}^{2} / \mathbb{Z}_{n}$. In section 3 , we study the Higgs branch of this theory. In section 4, we compute the 1-loop correction to the Coulomb branch metric. Section 5 is discussions.

\section{D1-D5 on $\mathbb{C}^{2} / \mathbb{Z}_{n}$}

In this section, we construct the Lagrangian on the D1-brane probe for the D5-branes on $\mathbb{C}^{2} / \mathbb{Z}_{n}$. 


\subsection{Symmetries}

To find the Lagrangian on the D1-brane, we first summarize the symmetries of the system. Let us consider a configuration of D1-branes and D5-branes extending in the $x^{0} x^{1}$ and $x^{0} x^{1} \cdots x^{5}$ directions, respectively. We can perform the orbifolding of the transverse directions $\mathbb{C}^{2}\left(=x^{6} x^{7} x^{8} x^{9}\right)$ by $\mathbb{Z}_{n}$ such that the resulting configuration is $1 / 8$ BPS. This can be seen by writing the BPS condition for the supersymmetry generator $\epsilon_{L} Q_{L}+\epsilon_{R} Q_{R}$

$$
\begin{aligned}
\text { D1 : } \epsilon_{L} & =\Gamma^{0} \Gamma^{1} \epsilon_{R} \\
\text { D5 }: \epsilon_{L} & =\Gamma^{0} \Gamma^{1} \Gamma^{2} \Gamma^{3} \Gamma^{4} \Gamma^{5} \epsilon_{R} \\
\text { KK }: \epsilon_{L} & =\Gamma^{0} \Gamma^{1} \Gamma^{2} \Gamma^{3} \Gamma^{4} \Gamma^{5} \epsilon_{L}, \quad \epsilon_{R}=\Gamma^{0} \Gamma^{1} \Gamma^{2} \Gamma^{3} \Gamma^{4} \Gamma^{5} \epsilon_{R}
\end{aligned}
$$

where $\epsilon_{L}, \epsilon_{R}$ are ten-dimensional Majorana-Weyl spinors with the same chirality. From these relations, it follows that the unbroken supersymmetry in the $x^{0} x^{1}$ space is chiral $\epsilon_{R}=\epsilon_{L}=\Gamma^{0} \Gamma^{1} \epsilon_{L}$. One can check that it is a two-dimensional $\mathcal{N}=(0,4)$.

Before orbifolding, the massless open string spectrum on the D1-brane is decomposed into various representations under the symmetry $S O(1,1)_{01} \times S O(4)_{2345} \times S O(4)_{6789}$. Following [14, we introduce the indices $\left(A^{\prime}, \widetilde{A}^{\prime}\right)$ and $(A, Y)$ to represent the fundamental 2 of various $S U(2)$ groups:

$$
\begin{aligned}
& S O(4)_{2345}=S U(2)_{A^{\prime}} \times S U(2)_{\widetilde{A}^{\prime}}, \\
& S O(4)_{6789}=S U(2)_{A} \times S U(2)_{Y} .
\end{aligned}
$$

The massless modes on the D1-brane coming from the 1-1 string and 1-5 string are summarized as 14 目

$$
\begin{array}{ccc} 
& b^{A Y} & \psi_{+}^{A^{\prime} Y} \\
1-1: & b^{A^{\prime} \widetilde{A}^{\prime}} & \psi_{+}^{A \widetilde{A}^{\prime}} \\
& A_{--}, A_{++} & \psi_{-}^{A A^{\prime}}, \psi_{-}^{\widetilde{A}^{\prime} Y} \\
1-5: & H^{A^{\prime}} & \chi_{+}^{A}, \chi_{-}^{Y}
\end{array}
$$

where $A_{ \pm \pm}=A_{0} \pm A_{1}$. This spectrum represent the $\mathcal{N}=(4,4) U\left(Q_{1}\right)$ gauge theory with one adjoint hypermultiplet and $Q_{5}$ fundamental hypermultiplets. $b^{A Y}$ is the scalar in the $\mathcal{N}=(4,4)$ vectormultiplet representing the position of D1-brane in the (6789) directions, while $b^{A^{\prime} \widetilde{A}^{\prime}}$ is the scalar in the adjoint hypermultiplet corresponding to the $(2345)$ directions. $S U(2)_{A^{\prime}}$ is usually denoted $S U(2)_{R}$ since the scalars in the hypermultiplets $b^{A^{\prime} \widetilde{A}^{\prime}}$

1 We flipped the $S O(1,1)$ chirality from [14] for later convenience. This is merely a convention of calling the remaining supersymmetry $(0,4)$ or $(4,0)$. 
and $H^{A^{\prime}}$ are doublets under this $S U(2)$. The $\mathcal{N}=(4,4)$ supersymmetry is generated by the supercharges

$$
Q_{+}^{A A^{\prime}}, \quad Q_{-}^{A^{\prime} Y}
$$

For example, the super transformation of $b^{A Y}$ is

$$
Q_{+}^{A A^{\prime}} b_{A}^{Y}=\psi_{+}^{A^{\prime} Y}, \quad Q_{-}^{A^{\prime} Y} b_{Y}^{A}=\psi_{-}^{A A^{\prime}} .
$$

Now we consider the orbifolding of $\mathbb{C}^{2}=\mathbb{R}_{6789}^{4}$ by $\mathbb{Z}_{n}$. We would like to preserve the right moving $(0,4)$ supersymmetry generated by $Q_{+}^{A A^{\prime}}$ and break the left moving one $Q_{-}^{A^{\prime} Y}$. Therefore, we embed the orbifold group $\mathbb{Z}_{n}$ in $S U(2)_{Y}$ :

$$
\mathbb{Z}_{n} \subset S U(2)_{Y} \subset S O(4)_{6789}
$$

Thus the nontrivial orbifold action is on the $Y$-index

$$
\left(b^{A Y}, \psi_{+}^{A^{\prime} Y}, \psi_{-}^{\widetilde{A}^{\prime} Y}, \chi_{-}^{Y}\right) \rightarrow\left(\omega^{Y} b^{A Y}, \omega^{Y} \psi_{+}^{A^{\prime} Y}, \omega^{Y} \psi_{-}^{\widetilde{A}^{\prime} Y}, \omega^{Y} \chi_{-}^{Y}\right)
$$

where $\omega=e^{2 \pi i / n}$ and $Y= \pm$, and the rest of the fields in (2.3) are invariant under the $\mathbb{Z}_{n}$.

To construct the gauge theory on the D1-brane, we follow the general procedure [1]. We extend the Chan-Paton factors $\mathcal{H}_{D 1}$ and $\mathcal{H}_{D 5}$ associated with the D1-branes and the D5-branes by tensoring $\mathbb{C}^{n}$. Physically this corresponds to going to the covering space of $\mathbb{C}^{2} / \mathbb{Z}_{n}$. Then we extend the orbifold action (2.7) by acting $\mathbb{Z}_{n}$ on the Chan-Paton factor $\mathbb{C}^{n}$ as well. We choose this action on $\mathbb{C}^{n}$ to be the regular representation of $\mathbb{Z}_{n}$. We also set $\mathcal{H}_{D 1}=\mathbb{C}$ and $\mathcal{H}_{D 5}=\mathbb{C}^{k}$, so that the gauge group becomes product of $U(1)$ 's.

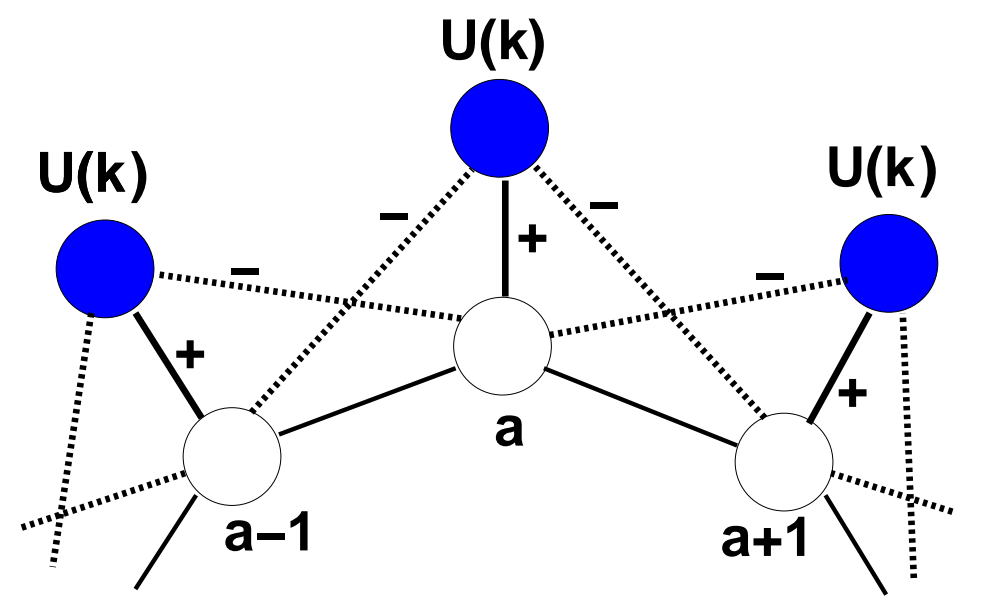

Fig. 1: The quiver diagram for D1-D5 on $\mathbb{C}^{2} / \mathbb{Z}_{n}$. The inner (outer) quiver corresponds to the D1-branes (D5-branes). The inner quiver is the $\widehat{A}_{n-1}$ Dynkin diagram (only three nodes are drawn in this figure). The links in the inner quiver represent a non-chiral $\mathcal{N}=(4,4)$ matter content, while the modes coming from the 1-5 string become chiral after orbifold projection. The dashed lines represent the left moving Fermi multiplets $\left(\Lambda_{a+1}^{Q f_{a}}, \Lambda_{f_{a+1}, a}^{\widetilde{Q}}\right)$. 
The resulting $\mathbb{Z}_{n}$ invariant spectrum are summarized by a quiver diagram (see Fig. 1). This is a two sets of $\widehat{A}_{n-1}$ Dynkin diagram connected by links. The inner (outer) quiver corresponds to the D1-brane (D5-brane) 目. The inner quiver represents the theory on the D1-branes on $\mathbb{C}^{2} / \mathbb{Z}_{n}$ constructed in [21], which is non-chiral and invariant under the $\mathcal{N}=(4,4)$ supersymmetry. This theory is well-known to lead to the hyperKähler quotient construction of $A_{n-1}$ ALE space. The left-right asymmetry comes from the orbifold action on the 1-5 string. As we can see, $H^{A^{\prime}}$ and $\chi_{+}^{A}$ in (2.3) are invariant under $\mathbb{Z}_{n}$ while $\chi_{-}^{Y}$ transforms non-trivially (2.7). This difference leads to the peculiar structure of the links connecting the inner and outer quivers as shown in Fig. 1.

\subsection{The Lagrangian in the $\mathcal{N}=(0,2)$ Language}

To write down the Lagrangian explicitly, it is useful to use the $\mathcal{N}=(0,2)$ superspace language. We follow the notation in $[17]^{3}$.

Before orbifolding, the theory representing the D1-D5 system is an $\mathcal{N}=(4,4) U\left(Q_{1}\right)$ gauge theory with one adjoint and $Q_{5}$ fundamental hypermultiplets. In the language of $\mathcal{N}=(2,2)$ superfields, the matter content of this theory is the vector multiplet $(\Sigma, \Phi)$, the adjoint hypermultiplet $(B, \widetilde{B})$ and the fundamental hypermultiplets $(Q, \widetilde{Q})$. All superfields are $\mathcal{N}=(2,2)$ chiral superfields, except for $\Sigma$ which is an $\mathcal{N}=(2,2)$ twisted chiral superfield. These fields correspond to the notation (2.3) in the previous section as

$$
b^{A Y} \leftrightarrow(\Sigma, \Phi), \quad b^{A^{\prime} \widetilde{A}^{\prime}} \leftrightarrow(B, \widetilde{B}), \quad H^{A^{\prime}} \leftrightarrow(Q, \widetilde{Q})
$$

To proceed, we need to know the orbifold action in the $\mathcal{N}=(2,2)$ language. Without loss of generality, we can choose the superspace coordinate $\theta^{-}$to carry the index $Y=+$. Therefore, the $\mathbb{Z}_{n}$ action in the $\mathcal{N}=(2,2)$ language is given by

$$
\begin{aligned}
& \Sigma\left(\theta^{+}, \bar{\theta}^{-}\right), \Phi\left(\theta^{+}, \theta^{-}\right) \rightarrow \omega \Sigma\left(\theta^{+}, \omega^{-1} \bar{\theta}^{-}\right), \omega^{-1} \Phi\left(\theta^{+}, \omega \theta^{-}\right) \\
& B\left(\theta^{+}, \theta^{-}\right), \widetilde{B}\left(\theta^{+}, \theta^{-}\right) \rightarrow B\left(\theta^{+}, \omega \theta^{-}\right), \widetilde{B}\left(\theta^{+}, \omega \theta^{-}\right) \\
& Q\left(\theta^{+}, \theta^{-}\right), \widetilde{Q}\left(\theta^{+}, \theta^{-}\right) \rightarrow Q\left(\theta^{+}, \omega \theta^{-}\right), \widetilde{Q}\left(\theta^{+}, \omega \theta^{-}\right)
\end{aligned}
$$

2 In Fig. 1, we didn't draw the links in the outer quiver, since they don't correspond to the dynamical fields on the D1-brane gauge theory.

3 See also [22,23] for useful reviews on $(0,2)$ linear sigma models. 
This condition can be written down in the $\mathcal{N}=(0,2)$ language by decomposing the $\mathcal{N}=(2,2)$ superfields into the $\mathcal{N}=(0,2)$ superfields:

$$
\begin{aligned}
& \Sigma_{(2,2)}\left(\theta^{+}, \bar{\theta}^{-}\right) \sim \Sigma-\sqrt{2} \bar{\theta}^{-} \Upsilon \\
& \Phi_{(2,2)}^{i}\left(\theta^{+}, \bar{\theta}^{-}\right) \sim \Phi^{i}+\sqrt{2} \theta^{-} \Lambda^{i}
\end{aligned}
$$

where $\Sigma$ and $\Phi^{i}$ are the $(0,2)$ chiral superfields, $\Upsilon$ is the $(0,2)$ gauge superfield, and $\Lambda^{i}$ is the Fermi superfield.

The resulting theory is an $\mathcal{N}=(0,4)$ gauge theory having $\prod_{a=1}^{n} U(1)_{a}$ as the gauge group and $\prod_{a=1}^{n} U(k)_{a}$ as the flavor symmetry. The kinetic term of the orbifolded theory is given by

$$
\begin{aligned}
& \mathcal{L}_{k i n}=-\frac{1}{2} \sum_{a=1}^{n} \int d \theta^{+} d \bar{\theta}^{+}\left[-\frac{i}{e^{2}} \bar{\Sigma}_{a+1, a} \mathcal{D}_{--} \Sigma_{a+1, a}-\frac{1}{e^{2}} \bar{\Upsilon}_{a} \Upsilon_{a}\right. \\
& +\frac{i}{e^{2}} \bar{\Phi}_{a, a+1} \mathcal{D}_{--} \Phi_{a, a+1}+\frac{1}{e^{2}} \bar{\Lambda}_{a}^{\Phi} \Lambda_{a}^{\Phi} \\
& +i \bar{B}_{a} \mathcal{D}_{--} B_{a}+\bar{\Lambda}_{a+1, a}^{B} \Lambda_{a+1, a}^{B} \\
& +i \overline{\widetilde{B}}_{a} \mathcal{D}_{--} \widetilde{B}_{a}+\bar{\Lambda}_{a+1, a}^{\widetilde{B}} \Lambda_{a+1, a}^{\widetilde{B}} \\
& +\sum_{f_{a}=1}^{k} i \bar{Q}_{f_{a} a} \mathcal{D}_{--} Q_{a}^{f_{a}}+\bar{\Lambda}_{f_{a}, a+1}^{Q} \Lambda_{a+1}^{Q f_{a}} \\
& \left.+i \overline{\widetilde{Q_{a}^{f_{a}}}} \mathcal{D}_{--} \widetilde{Q}_{f_{a} a}+\bar{\Lambda}_{a}^{\widetilde{Q}_{a+1}} \Lambda_{f_{a+1}, a}^{\widetilde{Q}}\right]
\end{aligned}
$$

and the $\mathcal{N}=(0,2)$ superpotential term is given by

$$
\begin{aligned}
\mathcal{L}_{p o t}=-\sum_{a=1}^{n} \int d \theta^{+}[ & \Lambda_{a+1, a}^{B}\left(\widetilde{B}_{a} \Phi_{a, a+1}-\Phi_{a, a+1} \widetilde{B}_{a+1}\right) \\
+ & \Lambda_{a+1, a}^{\widetilde{B}}\left(\Phi_{a, a+1} B_{a+1}-B_{a} \Phi_{a, a+1}\right) \\
+\sum_{f_{a}=1}^{k} & \left(\widetilde{Q}_{f_{a} a} \Phi_{a, a+1}-m_{f_{a}}^{f_{a+1}} \widetilde{Q}_{f_{a+1}, a+1}\right) \Lambda_{a+1}^{Q f_{a}} \\
+ & \Lambda_{f_{a+1}, a}^{\widetilde{Q}}\left(\Phi_{a, a+1} Q_{a+1}^{f_{a+1}}-Q_{a}^{f_{a}} m_{f_{a}}^{f_{a+1}}\right)+\widetilde{Q}_{f_{a} a} \Lambda_{a}^{\Phi} Q_{a}^{f_{a}} \\
& \left.+\left.\frac{1}{2} t_{a} \Upsilon_{a}\right|_{\left.\right|_{\bar{\theta}}=0}+\left.\frac{1}{2} s_{a} \Lambda_{a}^{\Phi}\right|_{\left.\right|^{+}=0}\right]+ \text { c.c.. }
\end{aligned}
$$

This $(0,2)$ superpotential is easily obtained by starting from the $(2,2)$ superpotential $W=\widetilde{Q}(\Phi-m) Q+\operatorname{tr} \widetilde{B}[\Phi, B]$, reducing to $(0,2)$ superspace, and then orbifolding by $\mathbb{Z}_{n}$. 
In (2.12), we introduced the FI-parameters and the theta-angle

$$
t_{a}=i \frac{\zeta_{a}^{3}}{e^{2}}+\frac{\theta_{a}}{2 \pi}, \quad s_{a}=\zeta_{a}^{1}+i \zeta_{a}^{2} .
$$

Let us explain our notation in (2.11) and (2.12). We use the same letter for the $(0,2)$ chiral superfields as the parent $(2,2)$ chiral superfields, and put the superscript in $\Lambda$ for the corresponding Fermi superfields. $\left(\Upsilon_{a}, \Lambda_{a}^{\Phi}, B_{a}, \widetilde{B}_{a}\right)$ are neutral multiplets living at the $a^{\text {th }}$ node in the inner quiver (see Fig. 1). $\left(\Sigma_{a+1, a}, \Lambda_{a+1, a}^{B}, \Lambda_{a+1, a}^{\widetilde{B}}\right)$ are living on the link connecting the $a^{\text {th }}$ node and the $(a+1)^{\text {th }}$ node in the inner quiver. They carry charge $(+1,-1)$ under the gauge group $U(1)_{a+1} \times U(1)_{a} . \Phi_{a, a+1}$ is living on the same link, but carries opposite charge $(-1,+1) \cdot Q_{a}^{f_{a}}$ and $\widetilde{Q}_{f_{a} a}$ are on the link between the $a^{\text {th }}$ node in the inner quiver and the $a^{\text {th }}$ node in the outer quiver; they transform as $(+1, \mathbf{k})$ and $(-1, \overline{\mathbf{k}})$ under $U(1)_{a} \times U(k)_{a}$, respectively. Finally, the Fermi multiplet $\Lambda_{a+1}^{Q f_{a}}$ is on the link between the $(a+1)^{\text {th }}$ inner node and the $a^{\text {th }}$ outer node, and $\Lambda_{f_{a+1}, a}^{\widetilde{Q}}$ is on the link between the $a^{\text {th }}$ inner node and the $(a+1)^{\text {th }}$ outer node; they transform as $(+1, \mathbf{k})$ and $(-1, \overline{\mathbf{k}})$ under $U(1)_{a+1} \times U(k)_{a}$ and $U(1)_{a} \times U(k)_{a+1}$, respectively.

The Fermi superfields are not chiral, but satisfy the constraint of the form $\overline{\mathcal{D}}_{+} \Lambda^{i}=$ $\sqrt{2} E^{i}$. In our case, the constraints are given by

$$
\begin{aligned}
\overline{\mathcal{D}}_{+} \Lambda_{a}^{\Phi} & =2\left(\Sigma_{a, a-1} \Phi_{a-1, a}-\Phi_{a, a+1} \Sigma_{a+1, a}-\mu_{a}\right) \\
\overline{\mathcal{D}}_{+} \Lambda_{a+1, a}^{B} & =2\left(\Sigma_{a+1, a} B_{a}-B_{a+1} \Sigma_{a+1, a}\right) \\
\overline{\mathcal{D}}_{+} \Lambda_{a+1, a}^{\widetilde{B}} & =2\left(\Sigma_{a+1, a} \widetilde{B}_{a}-\widetilde{B}_{a+1} \Sigma_{a+1, a}\right) \\
\overline{\mathcal{D}}_{+} \Lambda_{a+1}^{Q f_{a}} & =2\left(\Sigma_{a+1, a} Q_{a}^{f_{a}}-Q_{a+1}^{f_{a+1}} \widetilde{m}_{f_{a+1}}^{f_{a}}\right) \\
\overline{\mathcal{D}}_{+} \Lambda_{f_{a+1}, a}^{\widetilde{Q}} & =-2\left(\widetilde{Q}_{f_{a+1}, a+1} \Sigma_{a+1, a}-\widetilde{m}_{f_{a+1}}^{f_{a}} \widetilde{Q}_{f_{a}, a}\right) .
\end{aligned}
$$

In order for the $(0,2)$ superpotential $-\frac{1}{\sqrt{2}} \int d \theta^{+} \Lambda^{i} J_{i}$ to be chiral, $E^{i}$ and $J_{i}$ should be orthogonal [17]

$$
\frac{1}{\sqrt{2}} \overline{\mathcal{D}}_{+}\left(\Lambda^{i} J_{i}\right)=E^{i} J_{i}=0 \text {. }
$$

For our choice of the superpotential (2.12) and the constraint (2.14), the orthogonality (2.15) is satisfied by requiring

$$
m_{f_{a-1}}^{f_{a}} \widetilde{m}_{f_{a}^{\prime}}^{f_{a-1}}-\widetilde{m}_{f_{a+1}}^{f_{a}} m_{f_{a}^{\prime}}^{f_{a+1}}=\mu_{a} \delta_{f_{a}^{\prime}}^{f_{a}} .
$$

Note that $m_{f_{a}}^{f_{a+1}}$ and $\widetilde{m}_{f_{a+1}}^{f_{a}}$ correspond to the complex mass and the twisted mass, respectively, in the language of $\mathcal{N}=(2,2)$ theory. These parameters naturally live on the links of the outer quiver. 


\subsection{Potential Energy}

We expect that the theory flows in the IR to the non-linear sigma model with the target space given by the vanishing locus of the bosonic potential. There are three contributions to the potential energy: the $D$-term, the $E$-term, and the $J$-term

$$
U=\frac{1}{2 e^{2}} \sum_{a=1}^{n} D_{a}^{2}+\sum_{i}\left(\left|E^{i}\right|^{2}+\left|J_{i}\right|^{2}\right)
$$

The $E$-term and the $J$-term can be read off from (2.14) and (2.12). Note that the parameter $s_{a}$ in (2.12) enter in the $J$-term potential as

$$
2 e^{2} \sum_{a=1}^{n}\left|\widetilde{q}_{f_{a} a} q_{a}^{f_{a}}+\frac{1}{2} s_{a}\right|^{2} .
$$

Here and hereafter we denote the scalar component of $(0,2)$ chiral superfield by the lower case letter. The $D$-term for the $a^{\text {th }} U(1)$ gauge group is given by

$$
D_{a}=-e^{2}\left(\left|q_{a}^{f_{a}}\right|^{2}-\left|\widetilde{q}_{f_{a} a}\right|^{2}\right)+\left|\sigma_{a+1, a}\right|^{2}-\left|\phi_{a, a+1}\right|^{2}-\left|\sigma_{a, a-1}\right|^{2}+\left|\phi_{a-1, a}\right|^{2}+\zeta_{a}^{3} .
$$

In the following two sections, we will consider the moduli space of vacua defined by $U=0$.

\section{Higgs Branch}

In this section, we consider the Higgs branch, i.e. the vacuum with non-vanishing $q, \widetilde{q}$. For simplicity, we set $s_{a}=0$ and also set the mass term to be diagonal

$$
m_{f_{a}}^{f_{a+1}}=m_{a} \delta_{f_{a}}^{f_{a+1}}, \quad \widetilde{m}_{f_{a+1}}^{f_{a}}=\widetilde{m}_{a} \delta_{f_{a+1}}^{f_{a}} .
$$

This mass term breaks the flavor symmetry $\prod_{a=1}^{n} U(k)_{a}$ down to the diagonal $U(k)$. Then the parameter $\mu_{a}$ in (2.16) is given by

$$
\mu_{a}=m_{a-1} \widetilde{m}_{a-1}-m_{a} \widetilde{m}_{a}
$$

Instead of setting $D_{a}=E^{i}=J_{i}=0$, we can consider the holomorphic condition $E^{i}=J_{i}=0$ and mod out by the complexified gauge group $\prod_{a=1}^{n} \mathbb{C}_{a}^{\times}$. Then the equation for vacuum is given by

$$
\begin{aligned}
& \sigma_{a, a-1} \phi_{a-1, a}-\phi_{a, a+1} \sigma_{a+1, a}=m_{a-1} \widetilde{m}_{a-1}-m_{a} \widetilde{m}_{a} \\
& \phi_{a, a+1} q_{a+1}=m_{a} q_{a}, \quad \widetilde{q}_{a} \phi_{a, a+1}=m_{a} \widetilde{q}_{a+1} \\
& \sigma_{a+1, a} q_{a}=\widetilde{m}_{a} q_{a+1}, \quad \widetilde{q}_{a+1} \sigma_{a+1, a}=\widetilde{m}_{a} q_{a} \\
& \widetilde{q}_{a} q_{a}=0 .
\end{aligned}
$$


Here we suppressed the flavor index.

When all mass are non-zero, we can solve this equation up to the gauge transformation as

$$
\begin{aligned}
& \phi_{a, a+1}=m_{a}, \quad \sigma_{a+1, a}=\widetilde{m}_{a} \\
& q_{a}=q_{a+1}, \quad \widetilde{q}_{a}=\widetilde{q}_{a+1}, \quad \forall a
\end{aligned}
$$

Therefore, the conditions for $\left(q_{a}, \widetilde{q}_{a}\right)$ are reduced to the single equation $\widetilde{q}_{1} q_{1}=0$. It is known that the solution space of the equation $\widetilde{q}_{1} q_{1}=0$ modded out by $\mathbb{C}^{\times}$gives the $U(k)$ one-instanton moduli space on $\mathbb{R}^{4}$. Therefore, the Higgs branch is given by

$$
\mathcal{M}_{H}(m, \widetilde{m} \neq 0)=\mathcal{M}_{1, k}
$$

In the opposite extreme, i.e. when all mass are zero, the vacuum is given by $\sigma_{a+1, a}=$ $\phi_{a, a+1}=0$ and $\widetilde{q}_{a} q_{a}=0$. In this case, $q_{a}$ 's and $\widetilde{q}_{a}$ 's with different $a$ are unrelated. Therefore, the Higgs branch is given by

$$
\mathcal{M}_{H}(m=\widetilde{m}=0)=\left(\mathcal{M}_{1, k}\right)^{n} .
$$

We expect that in general the dimension of the Higgs branch jumps when setting some of the mass $m_{a}, \widetilde{m}_{a}$ to zero.

It is natural to expect that the boundary CFT of D1-D5-KK system is given by the Higgs branch CFT of D1-brane theory. It is interesting to observe that the Higgs branch of our $(0,4)$ theory is basically given by the instanton moduli space, which is the same as the Higgs branch of the D1-D5 system [2]. The similarity of the $(0,4)$ moduli space and the $(4,4)$ moduli space is emphasized in [9]. Our result (3.5), (3.6) is consistent with the conjecture [9] that the D1-D5-KK system admits a solvable boundary CFT written as a sigma model on the symmetric product of the manifold corresponding to the (2345) directions.

We should also mention that there is another branch $\mathcal{M}_{B}$ corresponding to the expectation value of $\left(B_{a}, \widetilde{B}_{a}\right)$. By a similar analysis as above, we find

$$
\begin{aligned}
\mathcal{M}_{B}(m, \widetilde{m} \neq 0) & =\mathbb{R}^{4} \\
\mathcal{M}_{B}(m=\widetilde{m}=0) & =\left(\mathbb{R}^{4}\right)^{n} .
\end{aligned}
$$

This branch represents the center-of-mass motion of D1-brane in the (2345) directions.

\footnotetext{
4 Turning on the FI-parameter corresponds to considering the instanton on non-commutative $\mathbb{R}^{4}$ [24].
} 


\section{Coulomb Branch}

In this section, we consider the Coulomb branch of our $(0,4)$ theory and compute the 1-loop correction to the effective metric. Let us first clarify our terminology of Coulomb and Higgs branch. The criterion is the Higgsing of the diagonal $U(1)$ of the gauge group $\prod_{a=1}^{n} U(1)_{a}$. In the branch with $q_{a}, \widetilde{q}_{a} \neq 0$ considered in the previous section, the diagonal $U(1)$ is broken. On the other hand, in the branch with $\sigma_{a+1, a}, \phi_{a, a+1} \neq 0$, the diagonal $U(1)$ is unbroken, so we will call this branch Coulomb branch.

Before going to the analysis of $(0,4)$ theory, we first review the $(4,4)$ case.

\subsection{Case 1: D1-D5}

Let us first consider the Coulomb branch of the system of single D1-brane and $Q_{5}$ D5-branes. The theory on the D1-brane is an $\mathcal{N}=(4,4) U(1)$ gauge theory with $Q_{5}$ hypermultiplets with charge 1 . The Coulomb branch is parametrized by the vectormultiplet $(\Sigma, \Phi)$. The metric on the Coulomb branch is corrected by integrating out the hypermultiplet. The 1-loop corrected metric is given by [25],3]

$$
d s^{2}=\left(\frac{1}{e^{2}}+\frac{Q_{5}}{|\phi|^{2}+|\sigma|^{2}}\right)\left(|d \phi|^{2}+|d \sigma|^{2}\right) .
$$

From the general structure of $\mathcal{N}=(4,4)$ non-linear sigma model, it can be shown that the metric is 1-loop exact. One can immediately notice that (4.1) is the metric on the transverse space of $Q_{5}$ NS5-branes [26]. Near the origin of Coulomb branch, this metric reduces to the familiar throat metric, and the CFT on the Coulomb branch is described by

$$
\mathcal{M}_{C}^{\text {near horizon }}=\mathbb{R}_{\phi} \times S U(2)_{Q_{5}}
$$

where $\mathbb{R}_{\phi}$ is the linear dilaton CFT and $S U(2)_{Q_{5}}$ is the WZW model.

\subsection{Case 2: D1 on $\mathbb{C}^{2} / \mathbb{Z}_{n}$}

The gauge theory on the D1-brane on $\mathbb{C}^{2} / \mathbb{Z}_{n}$ is given by the $\mathcal{N}=(4,4) \widehat{A}_{n-1}$ quiver gauge theory [1,21]. In our notation, this is given by the matter content on the inner quiver in Fig. 1. The fields written in the $(0,2)$ superspace naturally organize themselves into the $\mathcal{N}=(4,4)$ multiplets. Namely, $\left(B_{a}, \widetilde{B}_{a}, \Upsilon_{a}, \Lambda_{a}^{\Phi}\right)$ is the $(4,4)$ vectormultiplet living

on the $a^{\text {th }}$ node, and $\left(\Sigma_{a+1, a}, \Phi_{a, a+1}, \Lambda_{a+1, a}^{B}, \Lambda_{a+1, a}^{\widetilde{B}}\right)$ is the $(4,4)$ hypermultiplet living on the link between the $a^{\text {th }}$ node and the $(a+1)^{\text {th }}$ node. In this case, the diagonal $U(1)$ is 
decoupled and it never gets Higgsed. Although it is natural to call the branch of nonzero $\left(\sigma_{a+1, a}, \phi_{a, a+1}\right)$ Coulomb branch in the presence of D5-brane, this branch is usually referred to as the Higgs branch in the case of D1 on $\mathbb{C}^{2} / \mathbb{Z}_{n}$, since it is parametrized by the hypermultiplet.

It is known that the metric on the Higgs branch is the ALE metric. This is obtained by the following steps [27]. We first note that the action of the $(4,4)$ quiver theory is reproduced from our $(0,4)$ quiver theory by setting the fields from the 1-5 string to zero. In particular, the equations for the vacuum

$$
\begin{array}{r}
\sigma_{a, a-1} \phi_{a-1, a}-\sigma_{a+1, a} \phi_{a, a+1}=\mu_{a} \\
\left|\sigma_{a, a-1}\right|^{2}-\left|\phi_{a-1, a}\right|^{2}-\left|\sigma_{a+1, a}\right|^{2}+\left|\phi_{a, a+1}\right|^{2}=\zeta_{a}^{3} .
\end{array}
$$

is the same as those for the Coulomb branch of our $(0,4)$ theory. For the consistency, the FI-parameters have to satisfy the condition

$$
\sum_{a=1}^{n} \mu_{a}=\sum_{a=1}^{n} \zeta_{a}^{3}=0
$$

Next we introduce the variables $\left(\vec{r}_{a}, \varphi_{a}\right)$ by

$$
\begin{aligned}
2 \sigma_{a+1, a} \phi_{a, a+1} & =r_{a}^{1}+i r_{a}^{2} \\
\left|\sigma_{a+1, a}\right|^{2}-\left|\phi_{a, a+1}\right|^{2} & =r_{a}^{3} \\
\arg \left(\sigma_{a+1, a}\right) & =\varphi_{a} .
\end{aligned}
$$

The vacuum equations (4.3) imply that

$$
\vec{r}_{a}=\vec{r}-\vec{x}_{a}
$$

where $\vec{x}_{a}$ is given by

$$
\vec{x}_{a}=\sum_{b=1}^{a} \vec{\mu}_{b}, \quad \vec{\mu}_{a}=\left(2 \operatorname{Re} \mu_{a}, 2 \operatorname{Im} \mu_{a}, \zeta_{a}^{3}\right)
$$

Then we write down the kinetic term of $\left(\sigma_{a+1, a}, \phi_{a, a+1}\right)$ restricted on the locus 4.3

$$
\begin{aligned}
\mathcal{L}_{k i n} & =-\frac{1}{e^{2}} \sum_{a=1}^{n}\left[\left|D_{\mu} \sigma_{a+1, a}\right|^{2}+\left|D_{\mu} \phi_{a, a+1}\right|^{2}\right] \\
& =-\frac{1}{e^{2}} \sum_{a=1}^{n}\left[\frac{1}{\left|\vec{r}-\vec{x}_{a}\right|}\left(\partial_{\mu} \vec{r}\right)^{2}+\left|\vec{r}-\vec{x}_{a}\right|\left(\partial_{\mu} \varphi_{a}+\vec{\omega}_{a} \cdot \partial_{\mu} \vec{r}+A_{a, \mu}-A_{a+1, \mu}\right)^{2}\right]
\end{aligned}
$$


where $\vec{\omega}_{a}$ satisfies

$$
\nabla \times \vec{\omega}_{a}=\frac{1}{2} \nabla\left(\frac{1}{\left|\vec{r}-\vec{x}_{a}\right|}\right)
$$

Finally, by classically integrating out the gauge field $A_{a, \mu}$, we arrive at the effective Lagrangian on the locus 4.3 )

$$
\mathcal{L}_{e f f}=-\frac{1}{e^{2}}\left[V\left(\partial_{\mu} \vec{r}\right)^{2}+V^{-1}\left(\partial_{\mu} \theta+\vec{\omega} \cdot \partial_{\mu} \vec{r}\right)^{2}\right]
$$

where $V, \vec{\omega}$ and $\theta$ are given by

$$
V=\sum_{a=1}^{n} \frac{1}{\left|\vec{r}-\vec{x}_{a}\right|}, \quad \vec{\omega}=\sum_{a=1}^{n} \vec{\omega}_{a}, \quad \theta=\sum_{a=1}^{n} \varphi_{a} .
$$

The metric (4.10) is nothing but the familiar ALE metric. The above procedure is known as the hyperKähler quotient construction of ALE metric.

\subsection{Supergravity Analysis}

Now let us go back to the analysis of $(0,4)$ theory. From the discussion in the case 1,2 above, we can expect that the metric on the Coulomb branch of our $(0,4)$ theory reproduces the supergravity solution of D1-D5-KK system, which is given by the usual harmonic function rule [28]

$$
\begin{aligned}
d s^{2} & =\frac{1}{\sqrt{Z_{1} Z_{5}}}\left(-d t^{2}+d x_{1}^{2}\right)+\sqrt{\frac{Z_{1}}{Z_{5}}}\left(d x_{2}^{2}+\cdots d x_{5}^{2}\right)+\sqrt{Z_{1} Z_{5}} d s_{K K}^{2} \\
e^{-2 \Phi} & =\frac{Z_{5}}{Z_{1}}, \quad Z_{1,5}=1+\frac{Q_{1,5}}{r} .
\end{aligned}
$$

Here $d s_{K K}^{2}$ denotes the metric of KK-monopoles, i.e. Taub-NUT space. The metric seen by the probe D1-brane can be obtained by plugging the solution (4.12) into the D1-brane Born-Infeld action $S_{D 1}=-\int d \tau d \sigma e^{-\Phi} \sqrt{-\operatorname{det} g}$, and expanding it around the configuration $X^{0}=\tau, X^{1}=\sigma, X^{m}=X^{m}(\tau)$, where $X^{m}$ is the coordinate on the TaubNUT space. By looking at the term quadratic in the velocity $\dot{X}^{m}(\tau)$, we find that the effective metric seen by the D1-brane probe is

$$
d s_{\mathrm{D} 1-\text { probe }}^{2}=Z_{5} d s_{K K}^{2}
$$

This is independent of the factor $Z_{1}$ as expected from the BPS property. 
The metric (4.13) can also be obtained as the string metric on the S-dual F1-NS5-KK system. The S-dual of (4.12) is given by

$$
\begin{aligned}
& d s^{2 \prime}=e^{-\Phi} d s^{2}=\frac{1}{Z_{1}}\left(-d t^{2}+d x_{1}^{2}\right)+d x_{2}^{2}+\cdots d x_{5}^{2}+Z_{5} d s_{K K}^{2} \\
& e^{-2 \Phi^{\prime}}=e^{2 \Phi}=\frac{Z_{1}}{Z_{5}}
\end{aligned}
$$

Therefore, the metric on the transverse space seen by the fundamental string is

$$
d s_{\mathrm{NS} 5-\mathrm{KK}}^{2}=Z_{5} d s_{K K}^{2}
$$

which agrees with the D1-brane probe metric (4.13) as expected. In the next subsection, we compute the 1-loop correction to the metric on the Coulomb branch and compare it with the supergravity result (4.15).

\subsection{One-loop Correction to the Quiver Gauge Theory}

In this section, we compute the 1-loop correction to the metric on the Coulomb branch. There is no correction from $(B, \widetilde{B})$, since if we turn off the fields coming from the 1-5 string, the theory reduces to the $\mathcal{N}=(4,4)$ quiver gauge theory and it is known that there is no correction to the Higgs branch metric in this case. Recall that, as we discussed in section 4.2 , the role of Coulomb and Higgs branch is exchanged in the D1-brane on $\mathbb{C}^{2} / \mathbb{Z}_{n}$ case. Therefore, the 1-loop correction only comes from integrating out the modes of 1-5 string, i.e. the fields $\left(Q_{a}^{f_{a}}, \widetilde{Q}_{f_{a} a}, \Lambda_{a+1}^{Q f_{a}}, \Lambda_{f_{a+1}, a}^{\widetilde{Q}}\right)$. In this section, we set $m=\widetilde{m}=s_{a}=0$ for simplicity. Then the equation for vacuum is given by (4.3) with $\mu_{a}=0$.

Let us compute the 1-loop integral in the background of $\left(\sigma_{a+1, a}, \phi_{a, a+1}\right)$ obeying (4.3). The term quadratic in bosonic fields is given by

$$
\begin{aligned}
\mathcal{L}_{\text {boson }}=-\sum_{a=1}^{n} & \left(\left|D_{\mu} q_{a}\right|^{2}+2\left(\left|\sigma_{a+1, a}\right|^{2}+\left|\phi_{a-1, a}\right|^{2}\right)\left|q_{a}\right|^{2}\right. \\
& \left.+\left|D_{\mu} \widetilde{q_{a}}\right|^{2}+2\left(\left|\sigma_{a-1, a}\right|^{2}+\left|\phi_{a, a+1}\right|^{2}\right)\left|\widetilde{q}_{a}\right|^{2}\right)
\end{aligned}
$$

and the fermionic term is given by

$$
\mathcal{L}_{\text {fermi }}=\sum_{a=1}^{n} \Psi_{a}^{\dagger}\left(\begin{array}{cc}
i \partial_{--} & M_{a} \\
M_{a}^{\dagger} & i \partial_{++}
\end{array}\right) \Psi_{a}
$$


where $\Psi_{a}$ and $M_{a}$ are defined as

$$
\Psi_{a}=\left(\begin{array}{c}
\bar{\psi}_{+, a} \\
\widetilde{\psi}_{+, a} \\
\frac{\psi}{-, a+1}_{\widetilde{\psi}_{-, a-1}}
\end{array}\right), \quad M_{a}=\sqrt{2}\left(\begin{array}{cc}
-\bar{\sigma}_{a+1, a} & \bar{\phi}_{a-1, a} \\
\phi_{a, a+1} & \sigma_{a, a-1}
\end{array}\right)
$$

Here we suppressed the flavor indices for notational simplicity. We can check that the bosons and fermions have the same mass eigenvalues

$$
\begin{aligned}
M_{a} M_{a}^{\dagger} & =2\left(\begin{array}{cr}
\left|\sigma_{a+1, a}\right|^{2}+\left|\phi_{a-1, a}\right|^{2} & -\bar{\sigma}_{a+1, a} \bar{\phi}_{a, a+1}+\bar{\sigma}_{a, a-1} \bar{\phi}_{a-1, a} \\
-\sigma_{a+1, a} \phi_{a, a+1}+\sigma_{a, a-1} \phi_{a-1, a} & \left|\sigma_{a-1, a}\right|^{2}+\left|\phi_{a, a+1}\right|^{2}
\end{array}\right) \\
& =2\left(\begin{array}{cc}
\left|\sigma_{a+1, a}\right|^{2}+\left|\phi_{a-1, a}\right|^{2} & 0 \\
0 & \left|\sigma_{a-1, a}\right|^{2}+\left|\phi_{a, a+1}\right|^{2}
\end{array}\right) .
\end{aligned}
$$

In the last step, we used the vacuum equation (4.3) with $\mu_{a}=0$. Clearly, the eigenvalues of $M_{a} M_{a}^{\dagger}$ agree with the mass of $q_{a}, \widetilde{q}_{a}$.

Now we can compute the 1-loop effective action by integrating out the 1-5 string modes:

$$
\begin{aligned}
& \Gamma_{1-\text { loop }}=k \sum_{a=1}^{n}\left\{\log \operatorname{det}\left[\partial_{--} \partial_{++}+2\left(\left|\sigma_{a+1, a}\right|^{2}+\left|\phi_{a-1, a}\right|^{2}\right)\right]\right. \\
&+\log \operatorname{det}\left[\partial_{--} \partial_{++}+2\left(\left|\sigma_{a-1, a}\right|^{2}+\left|\phi_{a, a+1}\right|^{2}\right)\right] \\
&\left.-\log \operatorname{det}\left(\begin{array}{cc}
i \partial_{--} & M_{a} \\
M_{a}^{\dagger} & i \partial_{++}
\end{array}\right)\right\} \\
&=-k \sum_{a=1}^{n} \operatorname{Tr}\left[\frac{1}{\left(\partial_{--} \partial_{++}+M_{a} M_{a}^{\dagger}\right)^{2}} \partial_{++} M_{a} \partial_{--} M_{a}^{\dagger}\right]+\cdots
\end{aligned}
$$

Here we expanded the result up to two derivatives and the dots denote the higher derivative terms. The 1-loop integral in (4.20) can be evaluated by using the formula

$$
\int \frac{d^{2} p}{(2 \pi)^{2}} \frac{1}{\left(p^{2}+m^{2}\right)^{2}}=\frac{1}{4 \pi m^{2}} .
$$

Finally, we arrive at the 1-loop corrected effective Lagrangian

$$
\begin{aligned}
\mathcal{L}_{\mathrm{eff}}=-\sum_{a=1}^{n} & {\left[\frac{1}{e^{2}}\left(\left|D_{\mu} \sigma_{a+1, a}\right|^{2}+\left|D_{\mu} \phi_{a, a+1}\right|^{2}\right)\right.} \\
+ & \left.\frac{k}{4 \pi}\left(\frac{\left|D_{\mu} \sigma_{a+1, a}\right|^{2}+\left|D_{\mu} \phi_{a-1, a}\right|^{2}}{\left|\sigma_{a+1, a}\right|^{2}+\left|\phi_{a-1, a}\right|^{2}}+\frac{\left|D_{\mu} \sigma_{a, a-1}\right|^{2}+\left|D_{\mu} \phi_{a, a+1}\right|^{2}}{\left|\sigma_{a, a-1}\right|^{2}+\left|\phi_{a, a+1}\right|^{2}}\right)\right] .
\end{aligned}
$$


The first line in (4.22) is the tree level term and the second line is the 1-loop correction.

For the general value of the FI parameters $\zeta_{a}^{3}$, it is not so easy to rewrite this Lagrangian in terms of the variables $(\vec{r}, \theta)$ introduced in section 4.2. Here we focus on the orbifold limit corresponding to $\mu_{a}=\zeta_{a}^{3}=0$. Then we can show that

$$
\left|\sigma_{a+1, a}\right|^{2}+\left|\phi_{a-1, a}\right|^{2}=\left|\sigma_{a, a-1}\right|^{2}+\left|\phi_{a, a+1}\right|^{2}=|\vec{r}| \equiv r \quad \forall a
$$

This implies that the denominators in the second line in (4.22) are common for all terms and they can be factored out. Therefore, the 1-loop correction term in (4.22) becomes proportional to the tree level term. In other words, the metric on the Coulomb branch is given by the ALE metric up to a conformal factor:

$$
d s_{1-\mathrm{loop}}^{2}=\left(\frac{1}{e^{2}}+\frac{k}{2 \pi r}\right)\left(\frac{n}{r}(d \vec{r})^{2}+\frac{r}{n}(d \theta+\vec{\omega} \cdot d \vec{r})^{2}\right) .
$$

This is exactly the metric obtained in the supergravity approximation (4.15), with the understanding that the KK-monopole metric $d s_{K K}^{2}$ is replaced by the orbifold limit of the ALE metric. This shows that our $(0,4)$ theory can be thought of as a GLSM for the NS5-branes on ALE space. Near the origin of Coulomb branch, the metric (4.24) reduces to

$$
d s_{1-\mathrm{loop}}^{2} \sim \frac{k n}{2 \pi}\left[\frac{d r^{2}}{r^{2}}+d \Omega_{\mathbf{S}^{2}}^{2}+\frac{1}{n^{2}}(d \theta+\vec{\omega} \cdot d \vec{r})^{2}\right] .
$$

One can easily show that the last two terms is the metric on the lens space $\mathbf{S}^{3} / \mathbb{Z}_{n}$ written as the Hopf fibration of $\mathbf{S}^{1}$ over $\mathbf{S}^{2}$. Therefore, the near horizon limit is described by the $\mathbb{Z}_{n}$ orbifold of throat CFT

$$
\mathcal{M}_{C}^{\text {near horizon }}=\mathbb{R}_{\phi} \times \frac{S U(2)_{k n}}{\mathbb{Z}_{n}}
$$

where $\phi=\log r$ is the linear dilaton direction.

\section{Discussion}

In this paper, we have constructed the $\mathcal{N}=(0,4)$ quiver gauge theory corresponding to the D1-D5 branes on $\mathbb{C}^{2} / \mathbb{Z}_{n}$. The 1-loop correction of the Coulomb branch shows that this theory can be seen as a GLSM of NS5-branes on ALE space. Our result of 1-loop effective action on the Coulomb branch (4.22) is not proportional to the tree level term for the general FI parameters. This seems to suggest that the naive harmonic function 
rule breaks down in the string theory. We also expect that the effective metric for the $(0,4)$ theory is not 1-loop exact, although it is constrained by supersymmetry to be the hyperKähler with torsion sigma model. For both Higgs and Coulomb branch, our analysis is limited to the special value of the FI parameters. It would be interesting to study the general parameter case.

Acknowledgment: I would like to thank Yuji Sugawara for discussion in 1998. 


\section{References}

[1] M. R. Douglas and G. W. Moore, "D-branes, Quivers, and ALE Instantons," arXiv:hep-th/9603167.

[2] E. Witten, "On the conformal field theory of the Higgs branch," JHEP 9707, 003 (1997) arXiv:hep-th/9707093.

[3] D. E. Diaconescu and N. Seiberg, "The Coulomb branch of $(4,4)$ supersymmetric field theories in two dimensions," JHEP 9707, 001 (1997) arXiv:hep-th/9707158.

[4] N. Seiberg and E. Witten, "The D1/D5 system and singular CFT," JHEP 9904, 017 (1999) arXiv:hep-th/9903224.

[5] F. Larsen and E. J. Martinec, "U(1) charges and moduli in the D1-D5 system," JHEP 9906, 019 (1999) arXiv:hep-th/9905064.

[6] O. Aharony and M. Berkooz, "IR dynamics of $\mathrm{d}=2, \mathrm{~N}=(4,4)$ gauge theories and DLCQ of 'little string theories'," JHEP 9910, 030 (1999) arXiv:hep-th/9909101.

[7] D. Kutasov, F. Larsen and R. G. Leigh, "String theory in magnetic monopole backgrounds," Nucl. Phys. B 550, 183 (1999) [arXiv:hep-th/9812027.

[8] Y. Sugawara, "N = $(0,4)$ quiver $\operatorname{SCFT}(2)$ and supergravity on $\operatorname{AdS}(3)$ x S(2)," JHEP 9906, 035 (1999) arXiv:hep-th/9903120.

[9] F. Larsen and E. J. Martinec, "Currents and moduli in the $(4,0)$ theory," JHEP 9911, 002 (1999) arXiv:hep-th/9909088.

[10] I. Bena and P. Kraus, "Microstates of the D1-D5-KK system," Phys. Rev. D 72, 025007 (2005) arXiv:hep-th/0503053.

[11] A. Saxena, G. Potvin, S. Giusto and A. W. Peet, "Smooth geometries with four charges in four dimensions," arXiv:hep-th/0509214.

[12] J. M. Maldacena, A. Strominger and E. Witten, "Black hole entropy in M-theory," JHEP 9712, 002 (1997) arXiv:hep-th/9711053.

[13] R. Minasian, G. W. Moore and D. Tsimpis, "Calabi-Yau black holes and $(0,4)$ sigma models," Commun. Math. Phys. 209, 325 (2000) arXiv:hep-th/9904217.

[14] M. R. Douglas, "Gauge Fields and D-branes," J. Geom. Phys. 28, 255 (1998) arXiv:hep-th/9604198.

[15] E. Witten, "Sigma models and the ADHM construction of instantons," J. Geom. Phys. 15, 215 (1995) arXiv:hep-th/9410052.

[16] S. Gukov, E. Martinec, G. W. Moore and A. Strominger, "The search for a holographic dual to $\operatorname{AdS}(3) \times \mathrm{S}^{* *} 3 \times \mathrm{S}^{* *} 3 \times \mathrm{S}^{* *} 1$," arXiv:hep-th/0403090.

[17] E. Witten, "Phases of $\mathrm{N}=2$ theories in two dimensions," Nucl. Phys. B 403, 159 (1993) arXiv:hep-th/9301042.

[18] D. Tong, "NS5-branes, T-duality and worldsheet instantons," JHEP 0207, 013 (2002) arXiv:hep-th/0204186. 
[19] J. A. Harvey and S. Jensen, "Worldsheet instanton corrections to the Kaluza-Klein monopole," arXiv:hep-th/0507204.

[20] K. Okuyama, "Linear sigma models of H and KK monopoles," JHEP 0508, 089 (2005) arXiv:hep-th/0508097.

[21] C. V. Johnson and R. C. Myers, "Aspects of type IIB theory on ALE spaces," Phys. Rev. D 55, 6382 (1997) arXiv:hep-th/9610140.

[22] J. Distler, "Notes on (0,2) superconformal field theories," arXiv:hep-th/9502012.

[23] A. Adams, A. Basu and S. Sethi, "(0,2) duality," Adv. Theor. Math. Phys. 7, 865 (2004) arXiv:hep-th/0309226.

[24] N. Nekrasov and A. Schwarz, "Instantons on noncommutative $R^{* *} 4$ and $(2,0)$ superconformal six dimensional theory," Commun. Math. Phys. 198, 689 (1998) arXiv:hepth/9802068].

[25] M. R. Douglas, J. Polchinski and A. Strominger, "Probing five-dimensional black holes with D-branes," JHEP 9712, 003 (1997) arXiv:hep-th/9703031.

[26] C. G. . Callan, J. A. Harvey and A. Strominger, "Supersymmetric string solitons," arXiv:hep-th/9112030.

[27] G. W. Gibbons and P. Rychenkova, "HyperKaehler quotient construction of BPS monopole moduli spaces," Commun. Math. Phys. 186, 585 (1997) arXiv:hepth/9608085].

[28] C. V. Johnson, R. R. Khuri and R. C. Myers, "Entropy of 4D Extremal Black Holes," Phys. Lett. B 378, 78 (1996) arXiv:hep-th/9603061]. 